\title{
AVALIAÇÃO DO PAPEL DO ÓXIDO DE GRAFENO (GO) NA GERAÇÃO FOTOCATALÍTICA DE HIDROGÊNIO EM SISTEMAS BINÁRIOS (GO-CdS) E TERNÁRIOS (Pt-GO-CdS)
}

\author{
Cristiane Gomes Almeida ${ }^{a}$, Tuany Nascimento dos Santos Trindadea, Marcus Vinicius Santos da Silva e Luciana Almeida \\ Silva ${ }^{\mathrm{a}, \mathrm{b}, *}$ \\ anstituto de Química, Universidade Federal da Bahia, 40170-115 Salvador - BA, Brasil \\ 'Instituto Nacional de Ciência e Tecnologia de Energia e Ambiente (INCT), Universidade Federal da Bahia, $40170-290$ Salvador \\ - BA, Brasil \\ 'Instituto de Física, Universidade Federal da Bahia, 40170-115 Salvador - BA, Brasil
}

Recebido em 06/02/2018; aceito em 07/05/2018; publicado na web em 26/06/2018

\begin{abstract}
EVALUATION OF THE GRAPHENE OXIDE (GO) ROLE IN THE PHOTOCATALYTIC GENERATION OF HYDROGEN IN BINARY (GO-CdS) AND TERNARY (Pt-GO-CdS) SYSTEMS. Water splitting is a promising process to produce hydrogen from friendly feedstock and solar energy. In this work we have evaluated binary (GO-CdS) and ternary (Pt-GO-CdS) hybrid photocatalysts for hydrogen production assisted by visible light irradiation. Cadmium sulfide and composites with GO were prepared by sonochemical and thermal methods. GO addition took place by different strategies: during the synthesis or by mechanical mixture. A variety of configurations was tested and the best performance in hydrogen production among all materials was the ternary photocatalyst named $\mathrm{Pt}\left(\mathrm{GO} / \mathrm{CdS}_{\mathrm{TT}}\right)$, whose hydrogen production rate was $651 \mu \mathrm{mol} \mathrm{g} \mathrm{gat}^{-1} \mathrm{~h}^{-1}$. Such material was obtained by thermal method with GO addition during the synthesis. Additionally, the XRD and Raman analyses have confirmed the GO photoreduction during photocatalytic hydrogen evolution.
\end{abstract}

Keywords: water splitting; hydrogen; photocatalysis; graphene; cadmium sulfide; visible light.

\section{INTRODUÇÃO}

Recentemente, o hidrogênio tornou-se foco de uma verdadeira corrida científica no sentido de torná-lo, em definitivo, um vetor que irá compor a matriz energética em substituição aos derivados de petróleo. Diversas alternativas de produção já foram propostas e, constantemente, tecnologias de conversão de hidrogênio em diferentes formas de energia são aprimoradas, a exemplo das células a combustível. No entanto, ainda cerca de $95 \%$ do hidrogênio produzido são provenientes de combustíveis fósseis via reforma a vapor do gás natural. ${ }^{1,2}$ Neste cenário, é necessário o desenvolvimento de novas tecnologias que permitam produzir hidrogênio em larga escala utilizando fontes primárias renováveis e inesgotáveis, tais como energia solar, água e biomassa; de modo que as necessidades energéticas da humanidade sejam supridas com um combustível, de fato, limpo.

A decomposição direta da água em hidrogênio e oxigênio usando luz solar com auxílio de um semicondutor é um meio de imitar a fotossíntese das plantas e se configura como processo promissor com grande potencial de aplicação em larga escala. Esse processo é capaz de captar e distribuir energia solar armazenada na ligação H-H. O princípio da fotodecomposição da água já é bem conhecido desde a década de setenta quando Honda e Fujishima ${ }^{3}$ conseguiram produzir hidrogênio e oxigênio usando uma célula fotoeletroquímica, tendo $\mathrm{TiO}_{2}$ como fotoanodo e platina como fotocatodo. Uma partícula semicondutora com uma pequena quantidade de nanopartículas de platina depositada, ou outro metal adequado, é essencialmente uma célula fotoeletroquímica em miniatura, onde a água, ou outro doador de elétrons (hole scavenger), é oxidada na parte exposta do semicondutor (fotoanodo) e é reduzida na superfície das nanopartículas do metal (fotocatodo, que ainda atua como cocatalisador para a formação de hidrogênio molecular), resultando na decomposição fotocatalítica da água.

\footnotetext{
*e-mail: las@ufba.br
}

Sob irradiação com fótons de energia superior à energia de bandgap $\left(\mathrm{E}_{\mathrm{g}}\right.$, energia que separa a banda de valência $(\mathrm{BV})$ da banda de condução (BC) do semicondutor/fotocatalisador), elétrons na BV são excitados para a BC, gerando o par elétron-lacuna que participa de reações redox $\cdot{ }^{4-7}$ Para realizar a fotodecomposição da molécula de água, é necessário que o limiar da banda de condução do semicondutor esteja posicionado em potenciais mais negativos que o potencial de redução da $\mathrm{H}_{2} \mathrm{O}$ a $\mathrm{H}_{2}\left(\mathrm{E}_{\mathrm{r}}^{\circ}=-0,83 \mathrm{~V}\right.$ vs EPH em $\mathrm{pH}$ básico, ou $\mathrm{E}_{\mathrm{r}}^{\mathrm{o}}\left(\mathrm{H}^{+} / \mathrm{H}_{2}\right)=0 \mathrm{~V}$ em $\left.\mathrm{pH}=0\right)$, enquanto que o limiar da banda de valência deve estar posicionado em potenciais mais positivos que aquele de oxidação de $\mathrm{H}_{2} \mathrm{O}$ a $\mathrm{O}_{2}\left(\mathrm{E}_{\mathrm{r}}^{0}=+1,23 \mathrm{~V}\right.$ vs EPH, $\mathrm{pH}=0$ ou $\mathrm{E}_{\mathrm{r}}^{0}$ $\left(\mathrm{OH} / \mathrm{O}_{2}\right)=+0,40 \mathrm{~V}$ em pH básico). Termodinamicamente, a decomposição da água é uma reação não espontânea $\left(\Delta \mathrm{G}^{\circ}=+238 \mathrm{~kJ} \mathrm{~mol}^{-1}\right.$; $\Delta \mathrm{E}^{\mathrm{o}}=-1,23 \mathrm{~V}$ ), sendo necessário o suprimento de energia que coincide com fótons de energia de $\lambda \leq 1000 \mathrm{~nm}$ (i.e., $1,23 \mathrm{eV} \approx$ energia de fótons de $1000 \mathrm{~nm}$ ). Isto significa que a energia necessária para a produção fotocatalítica de hidrogênio a partir da água pode ser suprida pelo Sol.

Desde a década de 1980 o sulfeto de cádmio é um dos fotocatalisadores mais estudados na decomposição fotocatalítica da água sob irradiação de luz visível, ${ }^{8-20}$ que constitui a maior porção da radiação solar. Mas problemas associados à rápida recombinação de cargas fotogeradas, baixa estabilidade química em meio aquoso e os desafios no sentido de melhorar sua atividade fotocatalítica justificam o interesse constante em explorar esse material. ${ }^{21} \mathrm{O} \mathrm{CdS}$ é um semicondutor do tipo $n$, com energia de bandgap de $2,41 \mathrm{eV}$, portanto, ativo com luz visível, e potenciais de banda adequados para conduzir as reações de oxidação e redução da água $\left(\mathrm{E}_{\mathrm{BV}}^{\mathrm{o}}=+1,41 \mathrm{~V}\right.$ e $\left.\mathrm{E}_{\mathrm{BC}}^{\mathrm{o}}=-1,0 \mathrm{~V}\right)$. Em geral, problemas associados à fotocorrosão de CdS são minimizados com o emprego de doadores de elétrons, especialmente, os sistemas contendo o par $\mathrm{S}^{2-} / \mathrm{SO}_{3}{ }^{2-} \mathrm{em}$ meio básico, ${ }^{14}$ ou adição de um eletrólito quando o reagente de sacrifício é um álcool. ${ }^{22}$ Já problemas associados à recombinação de cargas são contornados com a introdução de átomos à rede de sulfeto formando soluções 
sólidas $^{23}$ ou com a associação a outro material em um compósito para a formação de heterojunção. ${ }^{24}$ Estudos apontam que sulfeto de cádmio em fase hexagonal apresenta maior atividade fotocatalítica na geração de hidrogênio comparada à fase cúbica. ${ }^{8,14,24,25}$ Geralmente, a fase hexagonal é obtida com o tratamento térmico de CdS comercial em atmosfera de nitrogênio e, recentemente, nós desenvolvemos um método sonoquímica para preparar nanoesferas de $\mathrm{CdS}$ hexagonal. ${ }^{2.5}$

Diversos metais podem ser empregados como fotocatodo na decomposição fotocatalítica da água, tais como Pt, Rh, Ru, Au, Pd e Ag, sendo a platina o mais usado. ${ }^{26,27}$ Nesses sistemas, os elétrons fotogerados com a excitação do semicondutor migram para as nanopartículas do metal, através da heterojunção, até o alinhamento dos níveis de Fermi, gerando a barreira Schottky na interface semicondutor/metal. ${ }^{28}$ A formação dessa barreira funciona como uma armadilha eletrônica impedindo a recombinação de cargas elétron-lacuna, evitando perda de energia na forma de calor. Nas reações de geração fotocatalítica de hidrogênio, os elétrons aprisionados são transferidos para prótons adsorvidos à superfície do metal que irão promover sua redução a hidrogênio molecular, enquanto que as lacunas fotogeradas no semicondutor irão oxidar a água ou um reagente de sacrifício. ${ }^{27}$ Deste modo, o semicondutor atua como anodo e o cocatalisador atua como catodo, semelhante a uma célula fotoeletroquímica.

Nos últimos anos, o grafeno tem despertado interesse de diversos grupos de pesquisa ao redor do mundo, inclusive os dedicados à produção fotocatalítica de hidrogênio, cujo objetivo é desenvolver metodologias capazes de obter compósitos de semicondutores com óxido de grafeno (GO) ou óxido de grafeno reduzido (RGO) de modo que estes desempenhem papel similar ao da platina ou atuem de forma sinérgica. ${ }^{29-41} \mathrm{O}$ grafeno tem grande área superficial específica e elevada mobilidade eletrônica $\left(10.000 \mathrm{~cm}^{2} \mathrm{~V}^{-1} \mathrm{~s}^{-1}\right),{ }^{35}$ propriedades que o tornam perfeito para atuar como aprisionador reversível e transportador de elétrons fotogerados em processos fotocatalíticos. Desta forma, é desejável que nesses sistemas prevaleça a forma reduzida (RGO), visto que a forma oxidada (GO) pode aprisionar irreversivelmente os elétrons fotogerados. ${ }^{36}$ Em geral, os compósitos semicondutor/ RGO são preparados a partir do GO obtido em etapas preliminares de oxidação do grafite e posterior esfoliação, com subsequente etapa de redução. A redução química é preferencialmente empregada que métodos alternativos, tais como térmico, eletroquímico e micro-ondas, por causar menos distorções e defeitos na regeneração da rede $\pi$ conjugada de átomo de carbono $\mathrm{sp}^{2} .^{37}$

Recentemente, diferentes estratégias vêm sendo testadas para preparar compósitos GO/CdS empregando diferentes métodos de síntese, tais como solvotérmico, coprecipitação e reação gás-sólido. ${ }^{36-41}$ No presente trabalho, foram empregados dois diferentes métodos de síntese, sonoquímico e térmico, para preparar compósitos de CdS hexagonal com óxido de grafeno, assim como foram feitas misturas mecânicas desses componentes, para avaliar a influência do método de preparação na atividade fotocatalítica para geração de hidrogênio em presença do par de agentes redutores $\mathrm{S}^{2-} / \mathrm{SO}_{3}{ }^{2-}$. A possibilidade de redução simultânea do óxido de grafeno, que elimina uma etapa prévia de redução no procedimento de preparo dos fotocatalisadores, também foi avaliada empregando técnicas de difratometria de raios $\mathrm{X}$ e espectroscopia Raman.

\section{PARTE EXPERIMENTAL}

\section{Materiais}

Os reagentes utilizados nas sínteses e testes fotocatalíticos são de grau analítico, exceto as soluções, e todos foram utilizados sem etapa prévia de purificação. Grafite, permanganato de potássio, sulfeto de sódio nonahidratado, etilenoglicol e soluções de ácido hexacloroplatínico $8 \%(\mathrm{~m} / \mathrm{m})$ e peróxido de hidrogênio $30 \%(\mathrm{~m} / \mathrm{m})$ foram adquiridos da Sigma Aldrich. Cloreto de cádmio, sulfeto de cádmio, sulfeto de sódio e tiossulfato de sódio foram adquiridos da Merck. O ácido sulfúrico usado foi da Qhemis, enquanto que brometo de cetiltrimetilamônio (CTBA) da VETEC.

\section{Síntese do óxido de grafite (GrO)}

O óxido de grafite foi preparado pelo método de Hummers modificado, que consiste na oxidação do grafite por permanganato de potássio, $\mathrm{KMnO}_{4}$, seguindo protocolo descrito por Mehl e colaboradores. ${ }^{42}$

\section{Síntese de CdS}

\section{Método sonoquímico}

O procedimento de síntese sonoquímica do sulfeto de cádmio hexagonal consistiu na dissolução de $0,9599 \mathrm{~g}$ de $\mathrm{CdCl}_{2}, 1,5055 \mathrm{~g}$ de $\mathrm{Na}_{2} \mathrm{~S}_{2} \mathrm{O}_{3}$ e $0,20 \mathrm{~g}$ de brometo de cetiltrimetilamônio (CTAB) em um béquer contendo $50 \mathrm{~mL}$ de etilenoglicol. Em seguida, a solução foi exposta à radiação ultrassônica usando uma sonda ultrassônica desruptora de célula R2D091109 da Unique, com os seguintes parâmetros: potência de $80 \mathrm{~W}$, ponteira de titânio diretamente imersa até cerca de $80 \%$ da mistura no béquer por 15 minutos. Ao final da reação, um precipitado amarelo foi obtido, separado por centrifugação e lavado, na sequência, duas vezes com água deionizada, duas vezes com etanol e seco à temperatura ambiente por 48 horas.

\section{Método térmico}

O sulfeto de cádmio em fase hexagonal também foi obtido por tratamento térmico do CdS comercial em atmosfera dinâmica de nitrogênio a $700{ }^{\circ} \mathrm{C}$ por $1 \mathrm{~h}$.

\section{Preparo dos compósitos}

Os compósitos foram preparados com adição de quantidades apropriadas de $\mathrm{GrO}$, de modo que o teor final de GO fosse de $2 \%$ (m/m), seguindo diferentes procedimentos; i) adição de $20 \mathrm{mg}$ de óxido de grafite $(\mathrm{GrO})$ à mistura reacional para obtenção de $1 \mathrm{~g}$ de sulfeto de cádmio sonoquímico, amostra denominada de GO/CdSson; ii) adição de $20 \mathrm{mg}$ de $\mathrm{GrO}$ a $1 \mathrm{~g}$ de $\mathrm{CdS}$ comercial em $50 \mathrm{~mL}$ de água e a suspensão sonicada por 15 minutos; em seguida, o sólido foi separado por centrifugação, seco e submetido a tratamento térmico em atmosfera de nitrogênio por $1 \mathrm{~h}$. Esta amostra foi denominada de $\mathrm{GO} / \mathrm{CdS}_{\mathrm{TT}}$; iii) misturas mecânicas de $\mathrm{GrO}$ com $\mathrm{CdS}$ obtido pelo método sonoquímico e $\mathrm{GrO}$ com $\mathrm{CdS}$ obtido pelo método térmico, seguidas de tratamento com radiação ultrassônica para esfoliação; amostras denominadas respectivamente de $\mathrm{GO}+\mathrm{CdS}$ son e $\mathrm{GO}+\mathrm{CdS}_{\mathrm{TT}}$. Nestes casos, $3 \mathrm{mg}$ de GrO, pesados em uma microbalança Mettler Toledo, modelo MX5, $\mathrm{d}=1 \mu \mathrm{g}$, foram misturados mecanicamente a $147 \mathrm{mg}$ de CdS; iv) adição de solução de $\mathrm{H}_{2}\left[\mathrm{PtCl}_{6}\right] \cdot 6 \mathrm{H}_{2} \mathrm{O} 8 \%(\mathrm{~m} / \mathrm{m})$ à mistura submetida ao teste fotocatalítico, em que $\mathrm{Pt}(\mathrm{IV})$ é fotorreduzida a $\mathrm{Pt}^{0}$ in situ. Nestes casos, as amostras foram denominadas de $\mathrm{Pt}(\mathrm{GO} / \mathrm{CdS}$ son $), \mathrm{Pt}(\mathrm{GO}+\mathrm{CdSson}), \mathrm{Pt}\left(\mathrm{GO} / \mathrm{CdS}_{\mathrm{TT}}\right)$ e $\mathrm{Pt}\left(\mathrm{GO}+\mathrm{CdS}_{\mathrm{TT}}\right)$, além das amostras sem GO, denominadas Pt/CdSson e Pt/CdS $\mathrm{S}_{\mathrm{TT}}$.

\section{Caracterização}

Todos os materiais preparados foram caracterizados por difratometria de raios $\mathrm{X}$, empregando um equipamento da Shimadzu modelo XRD 6000, radiação $\mathrm{CuK} \alpha$ e filtro de níquel. A velocidade de varredura programada foi de $2^{\circ} 2 \theta \min ^{1}$, na região $2 \theta$ de $5-80^{\circ}$, em $35 \mathrm{kV}$ e $15 \mathrm{~mA}$. Os difratogramas obtidos foram comparados com o banco de dados de padrões ICSD (Inorganic Crystal Structure 
Database) e dados da literatura para identificar as fases cristalinas das amostras. Os materiais ante e após irradiação foram caracterizados por Espectroscopia Raman, empregando um espectrômetro Raman dispersivo da Jasco NRS-5100, com resolução $0,4 \mathrm{~cm}^{-1}$.

\section{Testes fotocatalíticos}

Os testes fotocatalíticos foram conduzidos empregando $150 \mathrm{mg}$ do fotocatalisador em $100 \mathrm{~mL}$ de solução aquosa de $\mathrm{NaOH} 1 \mathrm{~mol} \mathrm{~L}^{-1}$, contendo $0,1 \mathrm{~mol} \mathrm{~L}^{-1}$ de $\mathrm{Na}_{2} \mathrm{~S} .9 \mathrm{H}_{2} \mathrm{O}$ e $0,02 \mathrm{~mol} \mathrm{~L}^{-1} \mathrm{de} \mathrm{Na}_{2} \mathrm{SO}_{3}$, adicionados a um reator com capacidade de $180 \mathrm{~mL}$. Nos testes contendo platina, esta foi fotodepositada in situ com adição do $40 \mu \mathrm{L}$ do precursor ácido hexacloroplatínico hexahidratado $\left(\mathrm{H}_{2}\left[\mathrm{PtCl}_{6}\right] \cdot 6 \mathrm{H}_{2} \mathrm{O}\right.$ $8 \% \mathrm{~m} / \mathrm{m})$. O reator empregado é equipado com entrada e saída de gás, conectadas em linha a um cromatógrafo para a retirada periódica de alíquotas da fase gasosa com auxílio de uma bomba de gás SCHARZER, modelo PN SP625EC. Antes do início da irradiação, para todos os sistemas, foi feita purga com argônio ultrapuro por $1 \mathrm{~h}$ a fim de eliminar o ar atmosférico para que a reação aconteça em meio anaeróbico. Oxigênio deve ser removido do meio, visto que este concorre com a água na reação com elétrons fotogerados para formar radicais superóxido. As reações fotocatalíticas foram conduzidas em atmosfera de argônio, à temperatura ambiente, com uma ventoinha posicionada na direção do reator para evitar variações significativas de temperatura. O reator foi irradiado com luz visível, empregando uma lâmpada de arco xenônio da Newport, com potência fixada em $500 \mathrm{~W}$ e área de exposição de $19,6 \mathrm{~cm}^{2}$. Para seleção da faixa espectral foram utilizados filtros de corte de radiação ultravioleta $(\lambda<400 \mathrm{~nm})$ e radiação infravermelha (filtro de água Newport) com refrigeração por meio de um banho termostático Quimis. Foram realizadas injeções no cromatógrafo de $1 \mathrm{em} 1 \mathrm{~h}$, em um total de 5 horas de irradiação. $\mathrm{O}$ hidrogênio molecular produzido nas reações fotocatalíticas foi analisado em um cromatógrafo a gás, Shimadzu CG-2014, com detecção de condutividade térmica (TCD), empregando coluna empacotada de peneira molecular, com as seguintes condições cromatográficas: argônio como gás de arraste a uma vazão de $10 \mathrm{~mL} \mathrm{~min}^{-1} \mathrm{e}$ temperatura de detecção de $200{ }^{\circ} \mathrm{C}$. Nestas condições, o tempo de retenção do hidrogênio é de 3 minutos, o qual foi quantificado por comparação com padrão de calibração de $5 \% \mathrm{H}_{2}$ em argônio.

\section{RESULTADOS E DISCUSSÃO}

A Figura 1 mostra os padrões de difração de raios $\mathrm{X}$ do grafite comercial (a) e dos materiais obtidos após sua oxidação (b) e esfoliação com tratamento ultrassônico por 15 minutos em água (c) e em solução aquosa de $\mathrm{S}^{2-} / \mathrm{SO}_{3}{ }^{2-}$ em meio básico (d), reproduzindo as condições de reação dos testes fotocatalíticos sem o fotocatalisador (CdS). Além disso, apresenta as amostras irradiadas com luz visível sem platina (e) e com platina (f) após o tratamento com radiação ultrassônica. $\mathrm{O}$ difratograma de raios $\mathrm{X}$ da amostra oxidada (b) apresenta o pico centrado em $26,68^{\circ}(2 \theta)$ referente ao conjunto de planos (002) do grafite, que sofre alargamento em função do processo de oxidação. Além desse pico, surge o pico em $10^{\circ}(2 \theta)$ associado ao conjunto de planos (002) do óxido de grafite (GrO). A esfoliação em água (c) resultou em um sólido com padrão de difração semelhante à amostra oxidada. O padrão de difração de raios X para o óxido de grafeno quando a esfoliação é bem sucedida apresenta um aumento na intensidade do pico em $10^{\circ}$ e o desaparecimento do pico referente ao grafite $\left(\sim 27^{\circ}\right)$, indicando que as lâminas do óxido de grafite sofreram desagregação para formar o GO..$^{42-44}$ Nesse trabalho, o tratamento com radiação ultrassônica em meio redutor resultou na formação de fases segregadas não identificadas, provavelmente de compostos de enxofre com o material carbonáceo. Quando irradiado na ausência de platina, surgem picos adicionais e aumento nas intensidades dos já existentes. Já quando o GrO é esfoliado e irradiado nas mesmas condições e com adição de platina, o número de picos referentes a fases segregadas diminui, assim como suas intensidades. O pico referente ao conjunto de planos (002) do grafite é regenerado e deslocado para $25,96^{\circ}(2 \theta)$, indicando aumento na distância interplanar, assim como o alargamento do pico que pode estar associado à diminuição do grau de empilhamento, resultando no agregado de um número pequeno de lâminas. Esses resultados constatam os já relatados, ${ }^{45,46}$ indicando que a redução fotoquímica do GO pode ocorrer mesmo sem o fotocatalisador, resultado da geração do par elétron-lacuna com a fotoexcitação do próprio GO. Os elétrons fotogerados atuam na redução do GO, enquanto que as lacunas oxidam o agente de sacrifício formado pelo par $\mathrm{S}^{2-} / \mathrm{SO}_{3}{ }^{2-}$.

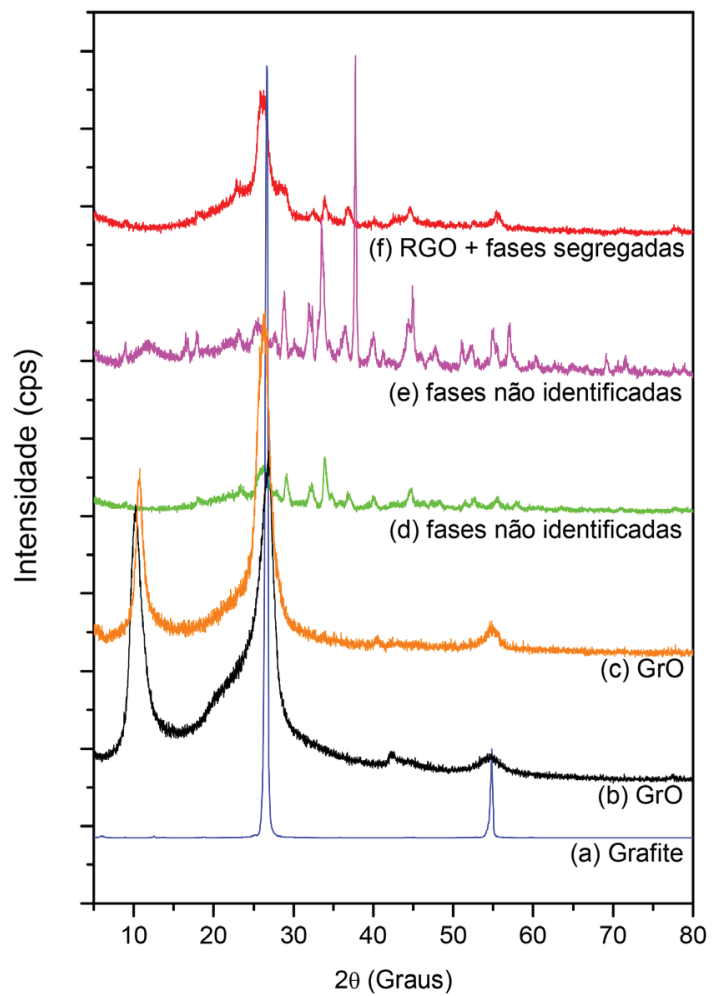

Figura 1. Difratogramas de raios $X$ das amostras de grafite (a), óxido de grafite, GrO, (b), GrO sonicado em água (c), GrO sonicado em solução aquosa contendo o par redox $\mathrm{S}^{2-} / \mathrm{SO}_{3}{ }^{2-}$ em meio básico (d), GrO esfoliado (em sol. $\mathrm{S}^{2-} / \mathrm{SO}_{3}{ }^{2-}$ ) e irradiado sem platina (e), $\mathrm{GrO}$ esfoliado (em sol. $\mathrm{S}^{2-} / \mathrm{SO}_{3}{ }^{2-}$ ) e irradiado em presença de platina $(f)$

Os difratogramas de raios $\mathrm{X}$ das amostras de $\mathrm{CdS}$ preparadas pelos métodos sonoquímico ( $\mathrm{CdSson})$ e térmico $\left(\mathrm{CdS}_{\mathrm{TT}}\right)$, assim como os dos compósitos GO/CdSson e GO/CdS $\mathrm{TT}_{\mathrm{TT}}$ são apresentados na Figura 2, acompanhados do padrão difração de $\mathrm{CdS}$ em fase hexagonal (pdf 01-075-1545-41-1049) para comparação. Em todos os casos é possível identificar os conjuntos de picos característicos dessa fase, porém, as amostras obtidas pelo método térmico apresentam picos estreitos e bem resolvidos, evidenciando elevada cristalinidade, característico de materiais obtidos por via térmica. Nos DRX das amostras de CdS térmico também é possível identificar uma fase segregada minoritária de CdO (pdf 01-073-2245-5-640), formada, provavelmente, devido à presença de traços de oxigênio durante o tratamento térmico em atmosfera de nitrogênio. Já os difratogramas das amostras de CdS obtidos pelo método sonoquímico não apresentam picos referentes à fase segregada, mas os picos associados à fase hexagonal são alargados em função da diminuição do tamanho dos cristalitos (uma 
discussão completa acerca da caracterização das nanopartículas de $\mathrm{CdS}$ obtidas por via sonoquímica pode ser encontrada na referência 25). Os difratogramas dos compósitos $\mathrm{GO} / \mathrm{CdS} s o n$ e $\mathrm{GO} / \mathrm{CdS}_{\mathrm{TT}}$ não apresentam diferenças significativas quando comparados aos de $\mathrm{CdS}$ puro obtido pelo respectivo método de síntese, não sendo possível identificar picos associados aos materiais carbonáceos tais como grafite, óxido de grafeno ou óxido de grafeno reduzido.

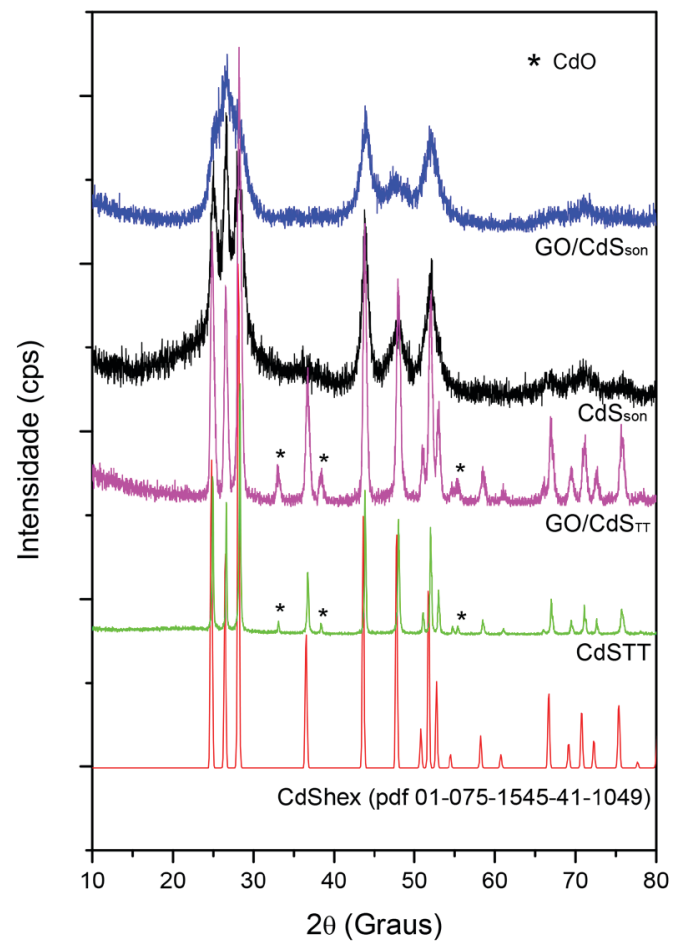

Figura 2. Difratograma de raios $X$ de CdS puro obtido pelos métodos sonoquímico (CdSson) e térmico ( $\left.C d S_{T T}\right)$ e na forma de compósitos com $\mathrm{GO}$ ( $\mathrm{GO}$ / CdSson e GO/CdS $S_{T T}$ ) e padrão de difração de CdS em fase hexagonal (pdf \# 01-075-1545-41-1049)

Os diâmetros médios de cristalitos das amostras de CdS puro e na forma de compósitos foram determinados a partir de dados de raios X. A equação de Scherrer (equação 1) foi empregada nos cálculos, considerando as partículas esféricas; onde $\mathrm{D}$ é o diâmetro médio dos cristalitos, $\lambda$ é o comprimento de onda dos raios X (1,541 Å), B é a largura a meia altura de um pico de difração em $2 \theta$.

$$
D=\frac{0,89 \lambda}{B \cos \theta}
$$

O pico centrado em torno de $43,8^{\circ}(2 \theta)$ foi selecionado para os cálculos por ser o de melhor resolução com maior intensidade nas quatro amostras. Os resultados encontram-se na Tabela 1 e constatam que o método sonoquímico é capaz de produzir cristalitos com diâmetro médio menor que o método térmico, como previsto. ${ }^{25}$ Além disso, a adição de GO às amostras de CdS durante a síntese contribui para redução do tamanho de partícula. No caso específico de $\mathrm{GO} / \mathrm{CdS}_{\mathrm{TT}}$, essa redução no diâmetro de cristalitos pode estar associada, também, à etapa de tratamento ultrassônico com o GO durante o preparo do compósito.

Todas as amostras foram empregadas como fotocatalisadores na reação de geração de hidrogênio sob irradiação de luz visível $(\lambda>$ $400 \mathrm{~nm}$ ), usando o sistema $\mathrm{S}^{2-} / \mathrm{SO}_{3}{ }^{2-}$ como reagentes de sacrifício em meio básico. Neste sistema, o sulfeto age como doador de elétrons, sendo oxidado a polissulfetos, enquanto o sulfito atua como agente
Tabela 1. Diâmetro de médio de cristalitos (D) de CdS puro e na forma de compósitos com GO, obtidos pelos métodos sonoquímico e térmico

\begin{tabular}{cc}
\hline Amostra & $\mathrm{D}(\mathrm{nm})$ \\
\hline CdSson & 10,9 \\
$\mathrm{GO} / \mathrm{CdSson}$ & 7,0 \\
$\mathrm{CdS}_{\mathrm{TT}}$ & 46,5 \\
$\mathrm{GO} / \mathrm{CdS}_{\mathrm{TT}}$ & 20,0 \\
\hline
\end{tabular}

regenerador dos íons sulfeto, indo a tiossulfato, de acordo com as equações 2 e $3 .{ }^{14}$ A regeneração do sulfeto por íons sulfito reestabelece a transparência da solução que se torna amarelada com a presença dos polissulfetos, já o meio básico evita a perda de sulfeto como $\mathrm{H}_{2} \mathrm{~S}$.

$\mathrm{nS}^{2-}+(2 \mathrm{n}-2) h^{+} \rightarrow \mathrm{S}_{\mathrm{n}}{ }^{2-}(\mathrm{n}=2-5) \quad$ (Solução amarela)

$2 \mathrm{~S}_{\mathrm{n}}{ }^{2-}+2(\mathrm{n}+1) / 3 \mathrm{SO}_{3}{ }^{2-}+3(\mathrm{n}-2) \mathrm{H}_{2} \mathrm{O} \rightarrow \mathrm{nS}_{2} \mathrm{O}_{3}{ }^{2-}+2(\mathrm{n}+1) / 3 \mathrm{~S}^{2-}+$ $2(\mathrm{n}-2) \mathrm{H}_{3} \mathrm{O}^{+}$(Solução transparente)

A Figura 3 mostra os perfis de evolução de hidrogênio em função do tempo de irradiação para as amostras com CdS hexagonal obtido pelo método sonoquímico (CdSson, GO/CdSson, GO+CdSson, $\mathrm{Pt}(\mathrm{GO} / \mathrm{CdSson}), \mathrm{Pt}(\mathrm{GO}+\mathrm{CdSson})$ e $\mathrm{Pt} / \mathrm{CdSon})$.

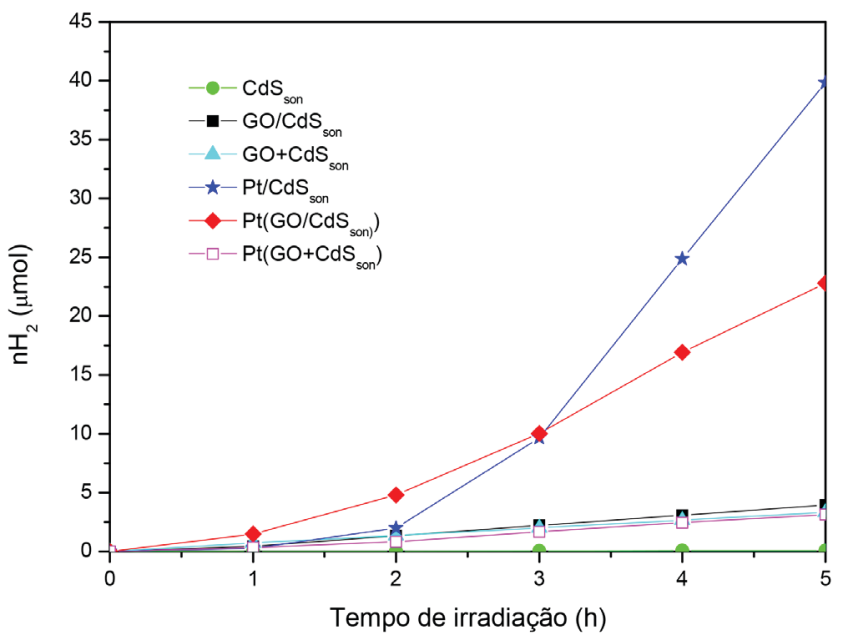

Figura 3. Produção fotocatalítica de hidrogênio $(\lambda>400 \mathrm{~nm})$ empregando CdS puro e compósitos com GO elou Pt em diferentes configurações obtidos pelo método sonoquímico

Pode-se notar que o CdS puro obtido pelo método sonoquímico apresenta atividade fotocatalítica negligenciável quando irradiado com luz visível na presença dos reagentes de sacrifício $\mathrm{S}^{2-} / \mathrm{SO}_{3}{ }^{2-}$. A adição do óxido de grafeno contribui para promover um pequeno aumento na atividade fotocatalítica dos sistemas binários $\mathrm{GO} / \mathrm{CdSson}$ e GO+CdSson, mas não há distinção entre os métodos de adição de GO na preparação dos compósitos. No entanto, o método de adição de GO é determinante na atividade fotocatalítica dos sistemas ternários $\mathrm{Pt}(\mathrm{GO} / \mathrm{CdSson})$ e $\mathrm{Pt}(\mathrm{GO}+\mathrm{CdSson})$, com um aumento significativo da atividade fotocatalítica quando GO é adicionado durante a síntese sonoquímica do CdS, o que proporciona sua maior dispersão no compósito. Das amostras avaliadas dentro desse grupo, a que apresentou maior atividade fotocatalítica foi a amostra $\mathrm{Pt} / \mathrm{CdSson}$, indicando que platina é um cocatalisador mais ativo que o GO nesse sistema e a introdução do óxido de grafeno na formação do compósito com CdS obtido pelo método sonoquímico não contribui para melhorar a fotoatividade na produção de hidrogênio. 
Os perfis de evolução de hidrogênio em função do tempo para as amostras contendo CdS hexagonal obtido pelo método térmico são mostrados na Figura 4. Os resultados revelam que a adição apenas de GO por mistura mecânica inibe a atividade fotocatalítica do $\mathrm{CdS}$ térmico (amostra $\mathrm{GO}+\mathrm{CdS}_{\mathrm{TT}}$ ), mas quando $\mathrm{GO}$ é disperso com radiação ultrassônica e tratado termicamente junto com $\mathrm{CdS}$ comercial $\left(\mathrm{GO} / \mathrm{CdS}_{\mathrm{TT}}\right)$ esta é melhorada. A adição de platina aumenta significativamente a atividade fotocatalítica de ambos, especialmente de $\mathrm{Pt}\left(\mathrm{GO} / \mathrm{CdS}_{\mathrm{TT}}\right)$ que tem a fotoatividade melhorada em mais de dez vezes quando comparada ao mesmo material sem a platina e em cerca de três vezes quando comparada ao material sem GO e com platina.

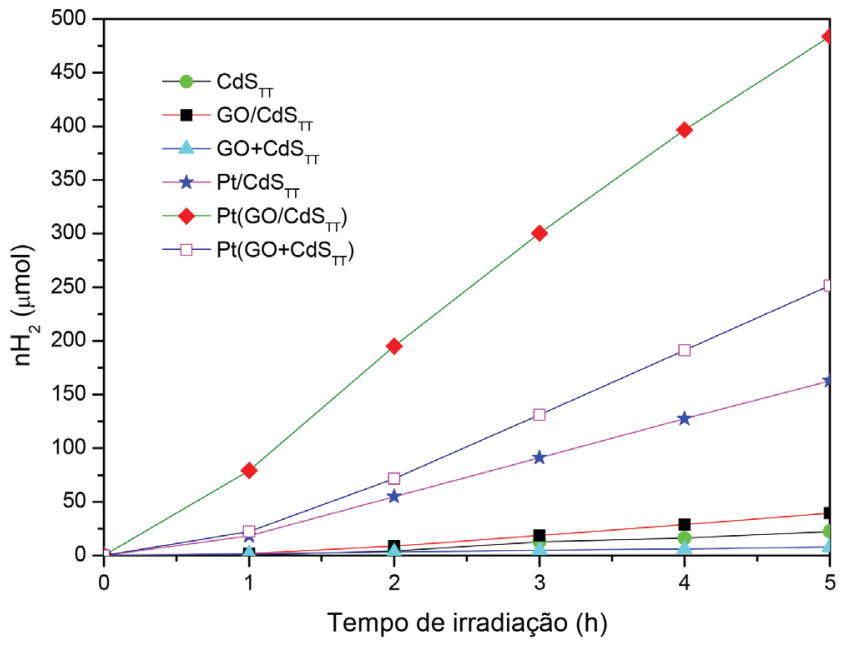

Figura 4. Produção fotocatalítica de hidrogênio $(\lambda>400 \mathrm{~nm})$ empregando CdS puro e compósitos com GO elou Pt em diferentes configurações obtidos pelo método térmico

A Tabela 2 compara as taxas de evolução de hidrogênio para amostras obtidas pelo método sonoquímico e pelo método térmico e revela que os fotocatalisadores obtidos pelo segundo método são mais ativos que aqueles obtidos pelo primeiro. Pode-se concluir que a maior fotoatividade na geração de hidrogênio está associada à maior cristalinidade dos materiais obtidos pelo método térmico, à maior dispersão do GO no compósito quando inserido durante a síntese e à presença da platina que atua como cocatalisador, além de auxiliar no processo de redução do óxido de grafeno como sugerem os resultados de difratometria de raios $\mathrm{X}$ apresentados na Figura 1. Sólidos mais cristalinos apresentam menor número de defeitos, geralmente, relacionados ao fenômeno de recombinação de cargas fotogeradas com a excitação do semicondutor, o que resulta em perda de energia na forma de calor. Esse fenômeno diminui a quantidade de portadores de carga e limita sua transferência na interface fotocatalisador/solução para promover as reações redox. A forma de adição do GO também

Tabela 2. Comparação entre as taxas de evolução de hidrogênio para amostras obtidas pelos métodos sonoquímico e térmico

\begin{tabular}{lcc}
\hline \multirow{2}{*}{ Fotocatalisador } & \multicolumn{2}{c}{$\mathrm{d}\left[\mathrm{H}_{2}\right] / \mathrm{dt}\left(\mu \mathrm{mol} \mathrm{g}_{\text {cat }}{ }^{-1} \mathrm{~h}^{-1}\right)$} \\
\cline { 2 - 3 } & Método sonoquímico & Método térmico \\
\hline $\mathrm{CdS}$ & 0,12 & 27,1 \\
$\mathrm{GO}+\mathrm{CdS}$ & 4,47 & 10,3 \\
$\mathrm{GO} / \mathrm{CdS}$ & 5,4 & 46,9 \\
$\mathrm{Pt} / \mathrm{CdS}$ & 53,4 & 209,0 \\
$\mathrm{Pt}(\mathrm{GO}+\mathrm{CdS})$ & 4,0 & 312,9 \\
$\mathrm{Pt}(\mathrm{GO} / \mathrm{CdS})$ & 31,3 & 651,6 \\
\hline
\end{tabular}

é um fator que interfere na fotoatividade, visto que a adição de GO durante o processo de síntese, em geral, resulta em maiores taxas de produção de hidrogênio quando comparada à mistura mecânica, provavelmente, devido à maior dispersão do GO no compósito.

A amostra $\mathrm{Pt}\left(\mathrm{GO} / \mathrm{CdS}_{\mathrm{TT}}\right)$ foi a que apresentou maior fotoatividade, com uma taxa de produção de hidrogênio de $651,6 \mu \mathrm{mol} \mathrm{g}_{\text {cat }}{ }^{-1}$ $\mathrm{h}^{-1}$. Esse resultado é superior aos obtidos por Leo e colaboradores ${ }^{36}$ e Singh e colaboradores ${ }^{37}$ que avaliaram a atividade fotocatalítica de compósitos $\mathrm{GO} / \mathrm{CdS}$ obtidos pelo método solvotérmico, seguido de redução química, e pelo método de reação gás-sólido, respectivamente, empregando o mesmo par de reagentes de sacrifício. Outros trabalhos ${ }^{38-41}$ apresentaram taxas de produção de hidrogênio superiores, especialmente quando o método solvotérmico é empregado na síntese dos fotocatalisadores; porém, nesses casos, foram feitas modificações químicas no GO, tais como inserção de dopantes, e outros reagentes de sacrifício foram usados.

Para avaliar a restauração da rede $\pi$ conjugada de átomos de carbono $s p^{2}$ pelo processo de fotorredução do óxido de grafeno (GO), os compósitos foram coletados ao final das 5 horas de irradiação para análise por difratometria de raios X e espectroscopia Raman e comparados com os materiais antes da irradiação.

Os fotocatalisadores pós irradiados foram submetidos à análise de difração de raios $\mathrm{X}$ para identificar picos referentes aos materiais carbonáceos. Nestes casos, as amostras pós irradiadas foram renomeadas substituindo GO por RGO, visto que há indícios de redução do óxido de grafeno durante as reações fotocatalíticas. A Figura 5 mostra os difratogramas de raios $\mathrm{X}$ das amostras de CdS e compósitos após irradiação e compara com as amostras de CdS puro e compósito $\mathrm{GO} / \mathrm{CdS}$ antes da irradiação para os dois métodos de síntese: (a) sonoquímico e (b) térmico. Os DRX das amostras GO/CdSson e $\mathrm{GO} / \mathrm{CdS}_{\mathrm{TT}}$ (antes da irradiação) apresentam apenas picos referentes ao CdS em fase hexagonal e é semelhante ao padrão de difração do CdS puro obtido pelo respectivo método de síntese, não sendo possível identificar o pico em $10^{\circ}(2 \theta)$ associado ao conjunto de planos (002) do GO. Entretanto, após irradiação, todas as amostras, exceto $\mathrm{RGO} / \mathrm{CdS}_{\mathrm{TT}}$ e $\mathrm{Pt}\left(\mathrm{RGO} / \mathrm{CdS}_{\mathrm{TT}}\right)$, apresentaram um pico largo em $21,04^{\circ}$ $(2 \theta)$, associado ao óxido de grafeno reduzido. ${ }^{42-44}$ A regeneração do pico referente ao conjunto de planos $(002)$ do grafite, originalmente em $26,68^{\circ}(2 \theta)$, agora deslocado para $21,04^{\circ}(2 \theta)$, indica um aumento significativo da distância interplanar, de 3,34 Å no grafite para 4,22 no óxido de grafeno reduzido presente nos compósitos após irradiação, assim como o alargamento do pico está associado a diminuição do grau de empilhamento das lâminas de grafeno. Os DRX das amostras $\mathrm{RGO} / \mathrm{CdS}_{\mathrm{TT}}$ e $\mathrm{Pt}\left(\mathrm{RGO} / \mathrm{CdS}_{\mathrm{TT}}\right)$, embora apresentem o pico referente ao conjunto de planos (002) deslocados para menor ângulo em relação grafite, o deslocamento foi menor comparado ao das demais amostras, assim como sua intensidade, provavelmente devido à maior agregação das lâminas, como consequência do tratamento térmico no processo de obtenção desses fotocatalisadores.

O processo de redução do óxido de grafeno também foi acompanhado por espectroscopia Raman. Esta técnica aplicada à análise do grafeno fornece informações únicas em termos estrutural, eletrônico e vibracional pelo fato da maioria das transições serem ressonantes. Essas transições no grafeno ocorrem entre as regiões dos pontos de alta simetria, $\Gamma, \mathrm{K}, \mathrm{M}, \mathrm{K}$ ', que limitam a chamada primeira zona de Brillouin, ${ }^{47,48}$ da célula unitária do grafeno. Além disso, as bandas de energia do material apresentam valores de mínimo e máximo em torno desses pontos, formando cones onde podem ocorrer diversas transições, tanto internas (intravale) quanto externas (intervale).

As medidas Raman foram utilizadas no monitoramento das intensidades da banda $\mathrm{G}\left(1585 \mathrm{~cm}^{-1}\right)$, associada a modos vibracionais de primeira ordem do fônon $\mathrm{E}_{2 \mathrm{~g}}$ dos átomos de carbono $s p^{2},{ }^{49,50}$ bem como da banda D $\left(1350 \mathrm{~cm}^{-1}\right)$, associada a defeitos da estrutura. Esta 

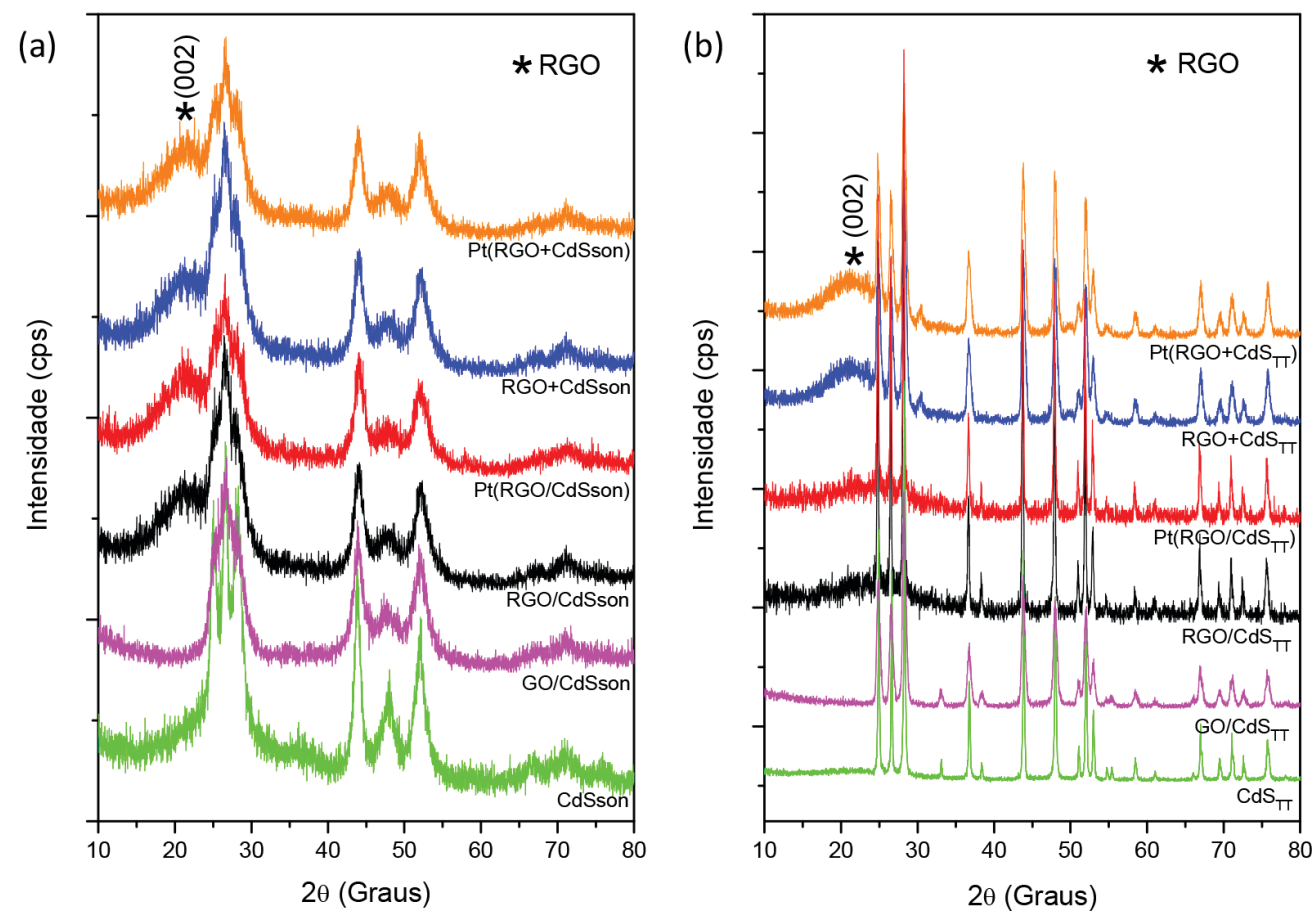

Figura 5. difratogramas de raios $X$ de CdS e compósitos, antes e depois da irradiação, obtidos pelos dois métodos de síntese: (a) sonoquímico e (b) térmico

última só é visível em estruturas carbonáceas com defeitos devido ao espalhamento inelástico e recombinação de portadores de carga excitados na rede..$^{48,51}$ No entanto, a medida absoluta dessas intensidades não é tarefa fácil. Por conta disso, a razão $\mathrm{I}_{\mathrm{D}} / \mathrm{I}_{\mathrm{G}}$ é largamente utilizada para avaliar o grau de desordem do material. ${ }^{48,52}$

De igual forma, foi possível monitorar a banda G' $\left(2718 \mathrm{~cm}^{-1}\right),{ }^{53}$ que apresenta dois picos (2D1 e 2D2) resultantes da interação de dois planos vizinhos, e da banda $\mathrm{G}^{*}\left(2430 \mathrm{~cm}^{-1}\right),{ }^{53}$ fruto de um processo intervale de segunda ordem originário de transições envolvendo um fônon longitudinal acústico (LA) e outro transversal óptico (iTO) na região do ponto $\Gamma$, centro da primeira zona de Brillouin. ${ }^{47}$

A Figura 6 compara os espectros dos compósitos preparados pelo método sonoquímico (a) e pelo método térmico (b), com introdução de GO no processo de síntese, antes e após a irradiação. O perfil dos espectros revela o efeito da irradiação no processo de redução do GO. Observa-se uma melhora na estrutura do material carbonáceo nos compósitos após irradiação, seja pela diminuição na razão $\mathrm{I}_{\mathrm{D}} / \mathrm{I}_{\mathrm{G}}$, seja

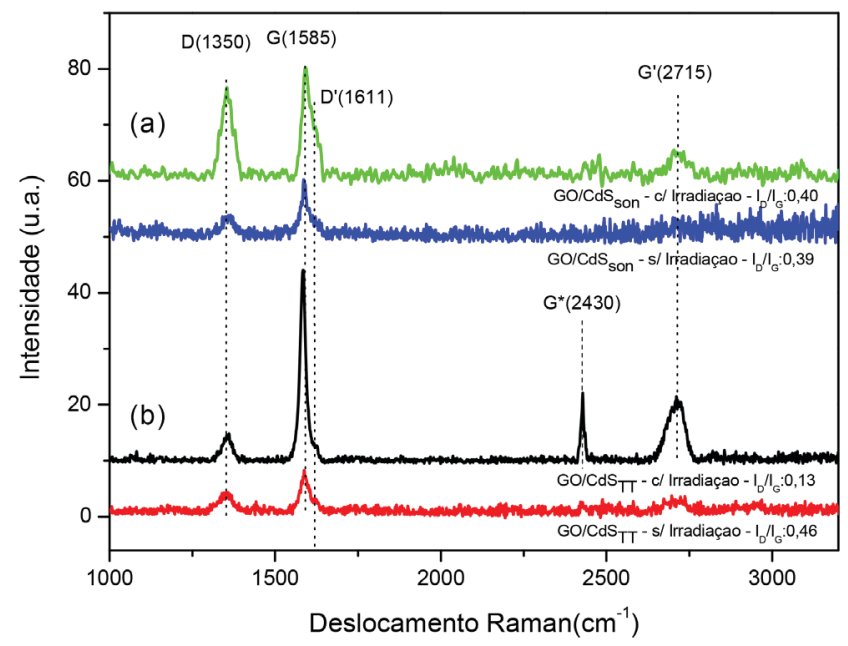

Figura 6. Espectros Raman dos compósitos GO/CdS, antes e após a irradiação, obtidos pelas vias sonoquímica (a) e térmica (b) pelo surgimento da banda G', indicando um menor grau de desordem. A melhor eficiência no processo de redução de GO é verificada para o compósito preparado pelo método térmico, no qual a razão $\mathrm{I}_{\mathrm{D}} / \mathrm{I}_{\mathrm{G}}$ diminui consideravelmente. No caso do compósito preparado pelo método sonoquímico, apesar do pequeno aumento da razão $\mathrm{I}_{\mathrm{D}} / \mathrm{I}_{\mathrm{G}}$, há também um aumento nas intensidades tanto da banda $\mathrm{G}$ quanto da $\mathrm{G}$ ' revelando também uma melhora estrutural do RGO nesse compósito.

Uma vez confirmado o efeito positivo da irradiação no processo de redução do GO, o passo seguinte foi comparar os espectros dos compósitos preparados pelo método sonoquímico (a) e pelo método térmico (b), com introdução de GO no processo de síntese ou por mistura mecânica nas diferentes configurações após irradiação, como mostra a Figura 7 (a e b).

Os espectros Raman (Figura 7) confirmam a redução do GO após irradiação nos demais compósitos produzidos com CdS pelas vias sonoquímica e térmica, conforme indicado anteriormente na Figura 6, bem como para os diferentes métodos de introdução do GO: reacional e mecânico. Os espectros que apresentam uma menor desordem são aqueles das amostras produzidas pelo método térmico (Figura 7b), em que são observadas menores razões $\mathrm{I}_{\mathrm{D}} / \mathrm{I}_{\mathrm{G}}$, tanto para a adição de GO durante à síntese quanto na mistura mecânica, corroborando os resultados de difração de raios $\mathrm{X}$ de uma melhor cristalinidade para esses compósitos. A banda G', associada à quantidade de camadas de grafeno, ${ }^{54,49}$ indica, em todos os espectros, estruturas de multicamadas, visto que a intensidade desta banda é bastante atenuada. No entanto, pela posição e forma do pico, há uma indicação de poucas camadas ( 3 - 5), confirmada pela pequena largura (FWHM) do pico $G^{*} .53,54$

Verificam-se, ainda nos espectros, dois picos de dupla ressonância: a transição D' $\left(\sim 1620 \mathrm{~cm}^{-1}\right),{ }^{53,55}$ relacionada ao processo intravale no cone do ponto $\mathrm{K}$, e que também está associada à desordem, ${ }^{53}$ mas com menor intensidade que a banda $\mathrm{D}$; e o pico $\mathrm{G}^{*}\left(\exp \sim 2430 \mathrm{~cm}^{-}\right.$ $\left.{ }^{1}\right),{ }^{56}$ que apresenta uma dispersão negativa, ${ }^{48,52}$ ou seja, a posição do pico se desloca para valores menores à medida que a energia do laser de excitação aumenta. ${ }^{57}$

Comparando os espectros Raman das amostras Pt(RGO/CdS $\left.\mathrm{TT}_{\mathrm{TT}}\right)$ e $\mathrm{Pt}\left(\mathrm{RGO}+\mathrm{CdS}_{\mathrm{TT}}\right)$, pós irradiadas, ambas obtidas pelo método térmico (Figura $7 \mathrm{~b}$ ), é possível observar que a razão $\mathrm{I}_{\mathrm{D}} / \mathrm{I}_{\mathrm{G}}$ menor e 

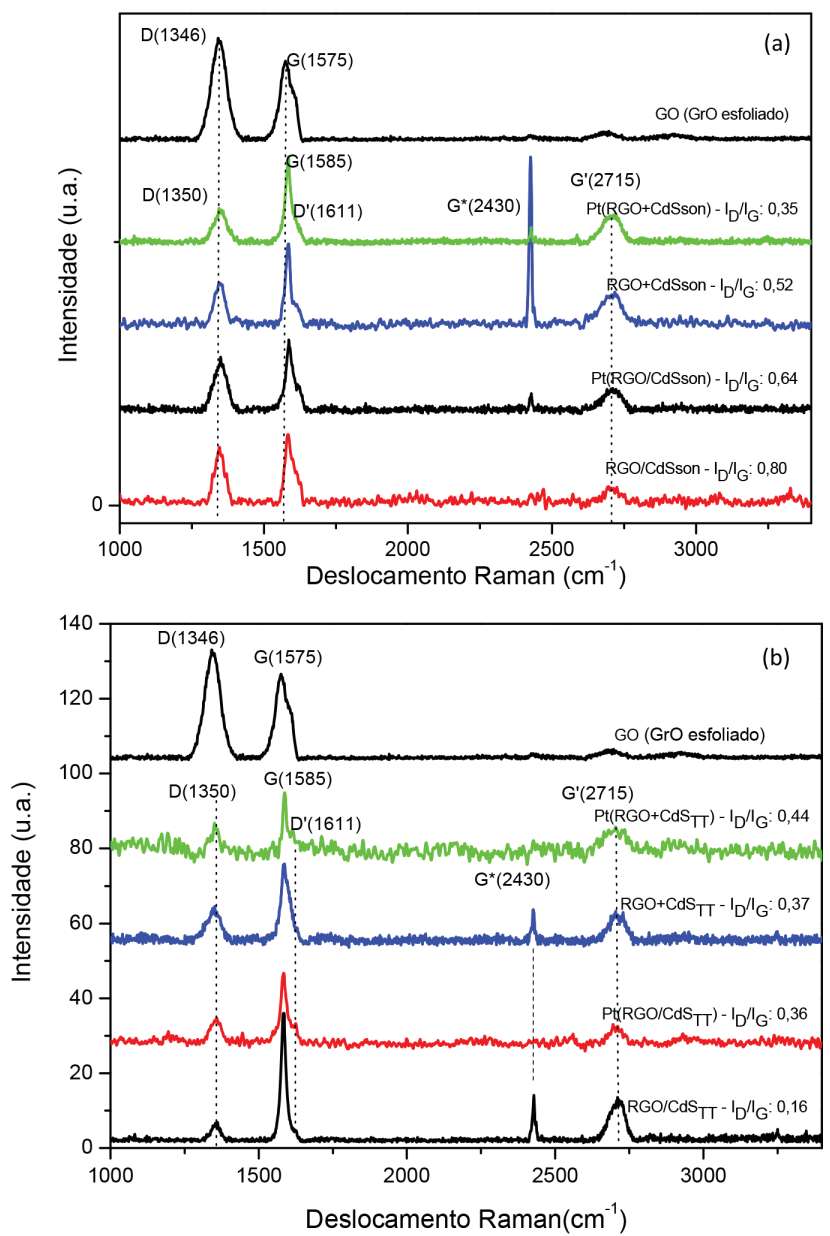

Figura 7. Espectros Raman do óxido de grafite esfoliado e dos compósitos de CdS pós irradiados obtidos por vias sonoquímica (a) e térmica (b)

a ausência da banda $\mathrm{G}^{*}$ no espectro da primeira representam uma melhor eficiência na redução do GO quando introduzido durante a síntese. Os resultados de análise por espectroscopia Raman indicam que o processo de fotorredução do óxido de grafeno é mais eficiente quando o compósito com CdS é obtido pelo método térmico comparado ao método sonoquímico, o que justifica maior atividade do fotocatalisador $\mathrm{Pt}\left(\mathrm{RGO} / \mathrm{CdS}_{\mathrm{TT}}\right)$ em relação aos demais.

Para o método térmico de síntese de CdS e compósitos, os sistemas ternários mostraram-se mais ativos que os binários, com destaque para $\mathrm{Pt}\left(\mathrm{RGO} / \mathrm{CdS}_{\mathrm{TT}}\right)$, fotocatalisador mais ativo dentre todos os testados nesse trabalho, indicando que platina e RGO, neste caso, atuam de forma sinérgica na geração fotocatalítica de hidrogênio, como ilustrado no esquema da Figura 8.

Durante a reação fotocatalítica tanto Pt(IV) quanto o óxido de grafeno são reduzidos, com possibilidade de fotodeposição de $\mathrm{Pt}^{0}$ na superfície de CdS e na do próprio RGO. O óxido de grafeno reduzido atua como aprisionador de elétrons que podem ser transferidos diretamente para moléculas de água adsorvidas à sua superfície ou podem ser transferidos para nanopartículas de platina que também promoverão a redução de $\mathrm{H}_{2} \mathrm{O}$ a $\mathrm{H}_{2}$. Além disso, a redução ainda pode se dar na superfície do CdS, na qual também ocorre a oxidação do sulfeto a polissulfetos pelas lacunas fotogeradas.

\section{CONCLUSÕES}

A atividade fotocatalítica dos compósitos de CdS com GO na geração de hidrogênio quando o par $\mathrm{S}^{2-} / \mathrm{SO}_{3}{ }^{2-}$ é usado como reagentes de sacrifício é dependente do método de síntese e da forma como GO

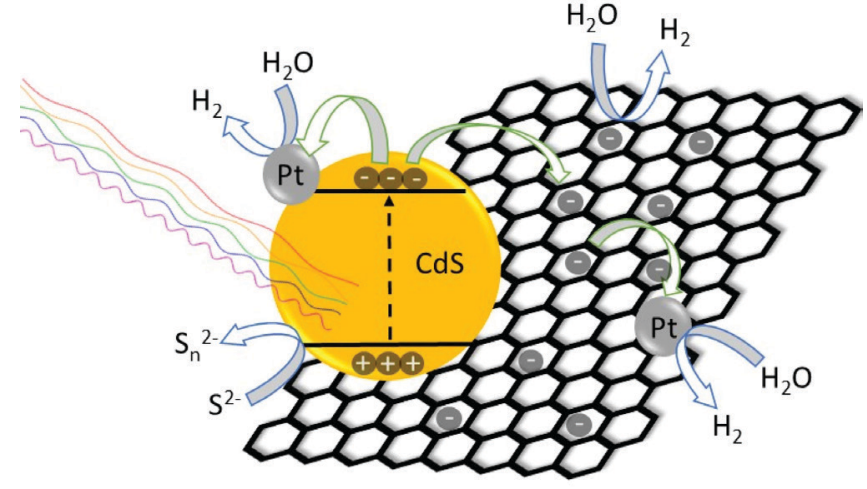

Figura 8. Ilustração dos processos primários de geração e transferência de carga no sistema ternário $\operatorname{Pt}\left(R G O / C d S_{T T}\right)$ para a produção fotocatalítica de hidrogênio

é introduzido. O método térmico de síntese resultou em compósitos mais fotoativos na geração de hidrogênio, especialmente se a platina está presente. Tanto as análises por difratometria de raios X quanto por espectroscopia Raman indicam que o óxido de grafeno nos compósitos é reduzido in situ durante a geração fotocatalítica de hidrogênio. Os resultados de DRX indicam que o RGO nos compósitos obtidos via rota sonoquímica apresenta maior distância interplanar e menor grau de empilhamento; já os resultados do estudo com espectroscopia Raman indicam que o RGO nos compósitos obtidos pelo método térmico apresenta menos defeitos e o processo de fotorredução do óxido de grafeno é mais eficiente quando comparado aos compósitos obtidos pelo método sonoquímico. Desta forma, o melhor desempenho do compósito $\mathrm{Pt}\left(\mathrm{RGO} / \mathrm{CdS}_{\mathrm{TT}}\right)$ está associado à maior cristalinidade do CdS hexagonal obtido pelo método térmico, a maior dispersão do GO quando introduzido durante a síntese, a maior eficiência no processo de fotorredução de GO a RGO em compósitos obtidos pelo método térmico e a atuação da platina como cocatalisador e auxiliar na fotorredução de GO. A combinação desses fatores resultou em um efeito sinérgico da platina com o óxido de grafeno reduzido, rendendo uma taxa de produção de hidrogênio de $651,6 \mu \mathrm{mol} \mathrm{g}_{\text {cat }}{ }^{-1} \mathrm{~h}^{-1}$.

\section{AGRADECIMENTOS}

Os autores são gratos ao CNPq pelo suporte financeiro (Processo 442840/2014-4), à CAPES pela bolsa de Doutorado concedida a C. G. Almeida e ao Laboratório Multi-Usuário de Microscopia Eletrônica da UFBA (LAMUME) pelas análises de espectroscopia Raman.

\section{REFERÊNCIAS}

1. Gallego, G. S.; Mondragón, F.; Barrault, J.; Tatibouët, J.-M.; BatiotDupeyrat, C.; Appl. Catal., A 2006, 311, 164.

2. Kappen, P.; Grunwaldt, J.-D.; Hammershoi, B. S.; Tröger, T.; Clausen, B. S.; J. Catal. 2001, 198, 56.

3. Fujishima, A.; Honda, K.; Nature 1972, 238, 37.

4. Hoffmann, M. R.; Martin, S. T.; Choi, W.; Bahnemann, D. W.; Chem. Rev. 1995, 95, 69.

5. Wang, C.; Pagel, R.; Dohrmann, J. K.; Bahnemann, D. W.; C. R. Chimie 2006, 9, 761.

6. Melo, M. O.; Silva, L. A.; J. Braz. Chem. Soc. 2011, 22, 1399.

7. Marques, F. C.; Stumbo, A. M.; Canela, M. C.; Quim. Nova 2017, 40, 561.

8. Matsumura, M.; Furukawa, S.; Saho, Y.; Tsubomura, H.; J. Phys. Chem. 1985, 89,1327

9. Matsumura, M.; Ohnishi, H.; Hanafusa, K.; Tsubomura, H.; Bull. Chem. Soc. Jpn. 1987, 60, 2001. 
10. Nosaka, Y.; Yamaguchi, K.; Kuwabara, A.; Miyama, H.; Baba, R.; Fujishima, A.; J. Photochem. Photobiol., A 1992, 64, 375.

11. Jin, Z. S.; Li, Q. L.; Zheng, X. H.; Xi, C. J.; Wang, C. P.; Zhang, H. Q.; Feng, L. B.; Wang, H. Q.; Chen, Z. S.; Jiang, Z. C.; J. Photochem. Photobiol., A 1993, 71, 85.

12. Janet, C. M.; Viswanath, R. P.; Nanotechnology 2006, 17, 5271.

13. Jang, J. S.; Li, W.; Oh, S. H.; Lee, J. S.; Chem. Phys. Lett. 2006, 425, 278.

14. Silva, L. A.; Ryu, S. Y. Choi, J.; Choi, W.; Hoffmann, M. R.; J. Phys. Chem. C. 2008, 112, 12069.

15. Souza E. A.; Silva, L. A.; J. Environ. Chem. Eng. 2016, 4, 2114.

16. Trindade, T. N. S.; Silva, L. A.; J. Alloys. Compd. 2018, 735, 400.

17. Rahmawati, F.; Yuliati, L.; Alaih, I. S.; Putri, F. R.; J. Environ. Chem. Eng. 2017, 5, 2251.

18. El-Maghrabi, H. H.; Barhoum, A.; Nada, A. A.; Moustafa, Y. M.; Seliman, S.; M.; Youssef, A. M.; Bechelany, M.; J. Photochem. Photobiol., A 2018, 351, 26

19. Chang, Y.; Yu, K.; Zhang, C.; Yang, Z.; Feng, Y.; Hao, H.; Jiang, Y.; Lou, L-L.; Zhou, W.; Liu, S.; Appl. Catal., B 2017, 215, 74.

20. Peng, Q.-X.; Xue, D.; Zhan, S.-Z.; Ni, C.-L.; Appl. Catal., B 2017, 219 , 353.

21. Ai, Z.; Zhao, G.; Zhong, Y.; Shao, Y.; Huang, B.; Wu, Y.; Hao, X.; Appl. Catal., B 2018, 221, 179.

22. Bastos, S. A. L.; Santos, F. N.; Lopes, P. A. L.; Silva; L. A.; Int. J. Hydrogen Energy 2014, 39, 14588.

23. Lopes, P. A. L.; Mascarenhas, A. J. S.; Silva; L. A.; J. Alloy. Compd. 2015, 649, 332.

24. Melo, M. O.; Silva, L. A.; J. Photochem. Photobiol., A 2011, 226, 36.

25. Lopes, P. A. L.; Santos, M. B.; Mascarenhas, A. J. S.; Silva, L. A.; Mater. Lett. 2014, 136, 111.

26. Acar, C.; Dincer, I.; Zamfirescu, C.; Int. J. Energy Res. 2014; 38, 1903.

27. Maeda, K.; J. Photochem. Photobiol., C 2011, 12, 237.

28. Wang, G.; Ling, Y.; Wang, H.; Lu, X.; Li, Y.; J. Photochem. Photobiol., C 2014, 19, 35.

29. Xue, C.; Yan, X.; An, H.; Li, H.; Wei, J.; Yang, G.; Appl. Catal., B 2018, 222, 157.

30. Chen, F.; Zhang, L.; Wang, X.; Zhang, R.; Appl. Surf. Sci. 2017, 422, 962.

31. Ali, M. B.; Jo, W.-K.; Elhouichet, H.; Boukherrou, R.; Int. J. Hydrogen Energy 2017, 42, 16449.

32. Kumar, D. P.; Hong, S.; Reddy, D. A.; Kim, T. K.; Appl. Catal., B 2017, 212,7

33. Siqi, L.; Zhang, C. Nan, Zhang; Zi-Rong, T.; Yi-Jun, X.; J Phys. Chem. C 2013; 117,8251 .
34. Li, X.; Shen, R.; Ma, S.; Chen, X.; Xie, J.; Appl. Surf. Sci. 2018, 430, 53.

35. Zhou, T.; Chen, F.; Liu, K.; Deng, H.; Zhang, Q.; Feng, J.; Fu, Q.; Nanotechnology 2011, 22, 045704.

36. Leo, M.; Soto, E.; Vaquero, F.; Mota, N.; Navarro, R. M.; Fierro, J. L. G.; Int. J. Hydrogen Energy 2017, 42, 13691.

37. Singh, S.; Sinha, A. S. K.; Appl. Surf. Sci. 2018, 430, 184.

38. Hong, Y.; Shi, P.; Wang, P.; Yao, W.; Int. J. Hydrogen Energy 2015, 40, 7045 .

39. Xu, J.; Wang, L.; Cao, X.; Chem. Eng. J. 2016, 283, 816.

40. Lei, Y.; Yang, C.; Hou, J.; Wang, F.; Min, S.; Ma, X.; Jin, Z.; Xu, J.; Lu, G.; Huang, K-W.; Appl. Catal., B 2017, 216, 59.

41. Khan, M. E.; Khan, M. M.; Cho, M. H.; J. Colloid Interface Sci. 2016, 482, 221.

42. Mehl, H.; Matos, C. F.; Neiva, E. G. C.; Domingues, S. H.; Zarbin, A. J. G.; Quim. Nova 2014, 37, 1639.

43. Stobinski, L.; Lesiak, B; Malolepszy, A.; Mazurkiewicz, M.; Mierzwa, B.; Zemek, J.; Jiricek, P.; Bieloshapk, I.; J. Electron Spectrosc. 2014, 195, 145.

44. Mauro, M.; Cipolletti, V.; Galimberti, M.; Longo, P.; Guerra, G.; J. Phys. Chem. C 2012, 116, 24809.

45. Cho, H-W.; Wu, J-J.; J. Colloid Interface Sci. 2015, 438, 291.

46. Li, X. H.; Chen, J. S.; Wang, X.; Schuster, M. E.; Schlogl, R.; Antonietti, M.; ChemSusChem 2012, 5, 642.

47. Dartora, C. A.; Jimenez, M. J. S.; Zanella, F.; Revista Brasileira de Ensino de Física, 2015, 37, 3301.

48. Childres, I.; Jauregui, L. A.; Park, W.; Cao, H.; Chen, Y. P. In New Developments in Photon and Materials Research; Jang, J. I., ed.; Nova Science Publishers: New York, 2013, Cap. 19.

49. Yoon, D.; Moon, H.; Cheong, H.; Choi, J. S.; Choi, J. A.; Park, B. H.; J. Korean Phys. Soc. 2009, 55, 1299.

50. Ferrari, A. C.; Solid State Commun. 2007, 143, 47.

51. Maultzsch, J.; Reich, S.; Thomsen, C.; Requardt, H.; Ordejon, P.; Phys. Rev. Lett. 2004, 92, 075501.

52. Dresselhaus, M. S.; Jorio, A.; Filho, A. G. S.; Saito, R.; Philos. Trans. R. Soc. 2010, 368, 5355.

53. Nemanich, R. J.; Solin, S. A.; Phys. Rev. B 1970, 15, 392.

54. Ferrari, A. C.; Meyer, J. C.; Scardaci, V.; Casiraghi, C.; Lazzeri, M.; Mauri, F.; Piscanec, S.; Jiang, D.; Novoselov, K. S.; Roth, S.; Geim, A. K.; Phys. Rev. Lett. 2006, 97, 187401

55. Ni, Z. H.; Wang, Y. Y.; Yu, T.; You, Y.; Shen, Z. X.; Phys. Rev. B 2008, 77, 235403

56. Ado, J.; International Scholarly Research Network, 2012, 1, 234216.

57. Popov, V. N.; J. Phys. Conf. Ser. 2016, 682, 012013. 